\title{
Comparison of Laparoscope-Assisted Single- Needle Laparoscopic Percutaneous Extraperitoneal Closure versus Open Repair for Pediatric Inguinal Hernia
}

\section{Shaofeng Wu}

Shanghai Children's Medical Center, Shanghai Jiaotong University School of Medicine

\section{Xiaoyu Xing}

Shanghai Children's Medical Center, Shanghai Jiaotong University School of Medicine

\section{Rong He}

Shanghai Children's Medical Center, Shanghai Jiaotong University School of Medicine

\section{Haiteng Zhao}

Shanghai Children's Medical Center, Shanghai Jiaotong University School of Medicine

\section{Liang Zhong}

Shanghai Children's Medical Center, Shanghai Jiaotong University School of Medicine Jie Sun ( $\nabla$ sunjie@scmc.com.cn )

Shanghai Children's Medical Center, Shanghai Jiaotong University School of Medicine

\section{Research Article}

Keywords: Inguinal hernia, Patent processus vaginalis, Laparoscopic hernia repair, Minimally invasive surgery, Children

Posted Date: December 16th, 2021

DOI: https://doi.org/10.21203/rs.3.rs-1156243/v1

License: (c) (i) This work is licensed under a Creative Commons Attribution 4.0 International License. Read Full License 


\section{Abstract \\ Background:}

Laparoscopic repairs have gained gradual acceptance in pediatric inguinal hernia over the past decade. However, consensus about the optimal management is still lacking. The aim of this study is to present a modified single-needle laparoscopic approach with the assistance of a laparoscope and to evaluate the surgical outcomes in comparison with traditional open repair method.

\section{Materials and Methods:}

We retrospectively reviewed the medical data of children who underwent laparoscope-assisted singleneedle laparoscopic percutaneous extraperitoneal closure (LPEC) and open repair (OR) for inguinal hernia from 2014 to 2019. Data were reviewed and analyzed with respect to the operating time, the incidence of metachronous contralateral inguinal hernia $(\mathrm{MClH})$, recurrence, and other complications.

\section{Results:}

In our cohort, 961 patients in the OR group and 1098 patients in the LPEC group were analyzed retrospectively. Mean operative time was significantly shorter in the LPEC group $(22.3 \pm 3.5 \mathrm{~min})$ than in the OR group $(27.8 \pm 5.9 \mathrm{~min})$ for bilateral hernia repair $(p<0.001)$. latrogenic cryptorchidism occurred statistically more frequently in the OR group than in the LPEC group $(0.4 \% \mathrm{vs.} 0 \%, \mathrm{p}=0.013)$. In addition, the incidence of $\mathrm{MCIH}$ was $3.7 \%(33 / 887)$ in the OR group and $0.3 \%(3 / 1014)$ in the LPEC group $(p<0.01)$.

\section{Conclusion:}

Based on the current outcomes, laparoscope-assisted single-needle LPEC provides a simple and attractive option for the administration of pediatric patients with inguinal hernia/hydrocele to reduce the $\mathrm{MClH}$.

\section{Introduction}

Inguinal hernias and hydroceles are common diseases in pediatric surgery, occurring in $5 \%$ of all neonates and almost 10\% of premature newborns [1]. Traditionally, inguinal hernia repair was performed with standard open repair via an inguinal crease incision. As laparoscopic procedures have become increasingly popular over the last 2 decades, this approach offers easy access to both internal rings for patency and potentially identifies non-clinically detected patency in patients [2]. Different maneuvers have been reported in laparoscopic repair for children with desirable success and comparable recurrence. Some comparative studies between laparoscopic hernia repair and conventional methods have also been reported, including a systematic review [3-5]. Few reports have focused on comparing the laparoscope- 
assisted single-needle LPEC with OR, especially in large cases. In the present study, we modified the surgical technique by using a single needle with laparoscope assistance to accomplish laparoscopic hernia repair. Except for dissection and ligation of the hernia sac using laparoscopic instruments, this approach can be performed with percutaneous suture ligation via the long tailor-made needle and the assistance of the laparoscope without additional laparoscopic ports.

The primary aim of this study was to present the details of the laparoscope-assisted single-needle LPEC and describe the results in comparison with open repair (OR) for over 2000 cases in one institution.

\section{Materials And Methods}

This was a retrospective cohort study, including 2059 patients who underwent inguinal hernia repair between 2014 and 2019 in a single institution. After ethics approval was obtained, medical data of patients who underwent open repair or laparoscope-assisted single-needle LPEC were reviewed. The diagnosis was made by the patient's medical history, physical examination, ultrasonography, or confirmed by visualization of the laparoscope during the surgery. Patients were excluded if they had undergone testicular surgery concomitantly or previously. Demographics, preoperative clinical presentations, intraoperative findings, complications, and follow-up information were reviewed and analyzed. Routine follow-up was performed regularly at 1 week for wound healing assessment, and 6, 12, and 24 months postoperatively to evaluate possible complications, in addition to testicular size and position for male patients. We also informed parents to revisit our outpatient clinic if they had any complaints after the regular follow-up period. Telephone interviews were performed for some patients at the 12- and 24-month time points.

\section{Surgical procedures}

\section{Open repair}

Open hernia ligation was performed by making a 1-3 cm inguinal incision. After the inguinal canal was dissected, the hernia sac was ligated highly after scrupulously freeing from the vas deferens and the vessels and then transected.

\section{Laparoscope-assisted single-needle LPEC}

Briefly, all patients were placed in the supine position, and surgery was performed under general anesthesia with tracheal intubation. First, pneumoperitoneum was established via a $5 \mathrm{~mm}$ trocar inserted through a small infra-umbilical incision using the open technique. On the hernia side, a $2 \mathrm{~mm}$ stab incision through the skin overlying the internal ring was made, and a 2-0 polyester suture was passed through the eyelet at the tip of the needle (20 cm length) (Fig. 1a). Then, the suture was passed along the medial aspect of the internal ring, elevating the peritoneum, dissecting the vas deferens and spermatic vessels off the peritoneum, and traversing over the vas deferens and spermatic vessels. Once the needle tip was advanced across the spermatic vessels, it was pierced through the peritoneum into the abdominal 
cavity, creating the first loop in the cavity. The needle was then removed completely from the abdomen after the suture loop was left behind with the assistance of the laparoscope to go through the loop (Fig. $1 \mathrm{~b}-\mathrm{d}$ ). Another suture was then re-introduced through the initial stab incision and along the lateral side of the internal ring, allowing the needle to advance into the abdomen at the previous puncture site. The preloaded loop with the needle was fed through the first loop and left in the abdomen with the assistance of the laparoscope, as previously described. The needle was removed totally from the abdomen and retreated off from the suture, and the first loop acted as a lasso by pulling the extra ends of the suture that created the purse-string circumferentially around the internal ring (Fig. 1e-g). Double ligation was then performed extracorporeally with care to ensure that the skin was free of the knot. Testis should be pulled downward softly and kept in a proper position in the scrotum. Fluid and air were to be evacuated manually from the scrotum or labia or aspirated by a needle with a syringe. A second look was performed to ensure complete ligation of the hernia (Fig. 1h). Prophylactic surgery was performed when the asymptomatic contralateral internal ring was confirmed of patency, and the same process was then repeated for the contralateral side.

\section{Statistical analysis}

Statistical analysis was performed using SPSS software (SPSS 22.0, Chicago, Illinois). Continuous data were analyzed by the Student's test. Categorical data were mainly analyzed using the chi-square test or Fisher's exact test. P-value was considered statistically significant when less than 0.05 .

\section{Results}

In our cohort, 961 patients in the OR group and 1098 patients in the LPEC group were analyzed retrospectively. All patient characteristics are presented in Table 1. The mean age at operation was $19.2 \pm 12.7$ months in the OR group and $17.5 \pm 18.4$ months in the LPEC group $(p=0.064)$, and mean body weight at operation was $12.9 \pm 7.4$ vs. $13.8 \pm 4.3 \mathrm{~kg}$ ( $(p=0.602)$, respectively. There was no statistically significant difference in patient age or weight between the two groups. 
Table 1

Preoperative patients characteristics in groups

\begin{tabular}{|llll|}
\hline & OR & LPEC & p value \\
\hline No. of patients & 961 & 1098 & 0.274 \\
\hline Male & $918(95.5 \%)$ & $1061(96.6 \%)$ & \\
\hline Female & $43(4.5 \%)$ & $37(3.4 \%)$ & \\
\hline Age (Months, SD) & $19.2 \pm 12.7$ & $17.5 \pm 18.4$ & 0.064 \\
\hline Weight (kg, SD) & $12.9 \pm 7.4$ & $13.8 \pm 4.3$ & 0.602 \\
\hline Laterals & & & 0.598 \\
\hline Right & $530(55.3 \%)$ & $632(57.5 \%)$ & \\
\hline Left & $357(37.1 \%)$ & $382(34.8 \%)$ & \\
\hline Bilateral & $74(7.7 \%)$ & $84(7.7 \%)$ & \\
\hline Mean follow-up (Months, SD) & $18.3 \pm 11.6$ & $16.7 \pm 15.7$ & 0.587 \\
\hline${ }^{*} p<0.05$ is statistically significant & & \\
\hline
\end{tabular}

\section{Table 2 Outcomes of procedure}

\begin{tabular}{|c|c|c|c|}
\hline & OR $(n=961)$ & LPEC $(n=1098)$ & $p$ value \\
\hline Intraoperative laterals & & & $<0.01^{*}$ \\
\hline unilateral & $887(92.3 \%)$ & $619(56.4 \%)$ & \\
\hline Bilateral & $74(7.7 \%)$ & $479(43.6 \%)$ & \\
\hline lateral ligation & 1035 & 1577 & \\
\hline Intraoperative cPPV & - & $39 \%(396 / 1014)$ & \\
\hline \multicolumn{4}{|l|}{ Surgical time (min, SD) } \\
\hline Unilateral & $15.4 \pm 6.1$ & $17.4 \pm 4.7$ & 0.178 \\
\hline Bilateral & $27.8 \pm 5.9$ & $22.3 \pm 3.5$ & $<0.01^{*}$ \\
\hline \multicolumn{4}{|c|}{$\mathrm{cPPV}=$ contralateral patency processus vaginalis } \\
\hline
\end{tabular}




\begin{tabular}{|llll|}
\hline \multicolumn{4}{|l|}{ Table 3 Comparison of the complications in groups } \\
\hline Bleeding/hematoma & $1.8 \%(19 / 1035)$ & $0.1 \%(2 / 1577)$ & $<0.01^{*}$ \\
\hline Wound infection & $1.1 \%(11 / 961)$ & $0.3 \%(3 / 1098)$ & 0.16 \\
\hline latrogenic cryptorchidism & $0.4 \%(4 / 984)$ & $0 / 1525$ & $0.013^{*}$ \\
\hline Testicular atrophy & $0.2 \%(2 / 984)$ & $0 / 1525$ & 0.078 \\
\hline Recurrence & $1.3 \%(13 / 1035)$ & $0.5 \%(6 / 1182)$ & 0.056 \\
\hline MCIH & $3.7 \%(33 / 887)$ & $0.3 \%(3 / 1014)$ & $<0.01^{*}$ \\
\hline$M C I H:$ Metachronous Contralateral Inguinal Hernia & \\
\hline${ }^{*} p<0.05$ is statistically significant & & \\
\hline
\end{tabular}

Of all 2059 patients, a total of 1035 hernia rings (887 unilateral and 74 bilateral) were ligated in the 961 patients in the OR group (Table 2). However, in the LPEC group, 1014 of 1098 patients were clinically diagnosed with unilateral hernia (632 right-sided and 382 left-sided inguinal hernia), and 39\% (396/1014) of them were confirmed to have asymptomatic contralateral PPV intraoperatively and underwent prophylactic surgery. Hence, in the LPEC group, 619 of 1098 patients underwent unilateral surgery and 479 of 1098 underwent bilateral surgery. A total of 1577 internal inguinal rings (619 unilateral and 479 bilateral, including 396 contralateral PPVs) were ligated in the 1098 patients in the LPEC group (Table 2). Operative time was compared between the two groups, mean operative time for unilateral surgery in the OR and LPEC groups were $15.4 \pm 6.1 \mathrm{~min}$ and $17.4 \pm 4.7 \mathrm{~min}$, respectively. The difference was not statistically significant in unilateral repair $(p=0.178)$, while the operative time was significantly shorter in the LPEC group $(22.3 \pm 3.5 \mathrm{~min})$ than in the OR group $(27.8 \pm 5.9 \mathrm{~min})$ for bilateral repair $(p<0.01$, Table 2$)$.

A comparison of complications between the OR and LPEC groups are shown in Table 3. All LPEC administrations were performed without open conversion or requiring additional skin incision. For 8 male patients whose hernia contained omentum adhesive to the hernia sac, another $3 \mathrm{~mm}$ forceps were introduced through the extended infraumbilical incision to retract to the abdomen. There were no injuries observed to any intra-abdominal organs except in cases of bleeding or hematoma. Inguinal bleeding or hematoma occurred more frequently in the OR group $(1.8 \% ; 19 / 1035)$ than in the LPEC group $(0.1 \%$; $2 / 1577)(p<0.01)$. All bleeding or hematoma was observed regularly or controlled by external compression at the groin site. In the OR group, wound infections were recorded in 11 cases $(1.1 \%)$, whereas in the LPEC group, only 3 cases $(0.3 \%)$ developed. There were no statistically significant differences between the two groups regarding wound infection that needed further treatment $(p=0.16)$.

The mean follow-up period was $18.3 \pm 11.6$ months in the OR group and $16.7 \pm 15.7$ months in the LPEC group ( $p=0.587$ ). The total follow-up rates were $91.3 \%$ and $84.7 \%$ at 12 and 24 months, respectively. 
During this period, iatrogenic cryptorchidism occurred statistically more frequently in the OR group ( $0.4 \%$; $4 / 984)$ than in the LPEC group $(0 \% ; 0 / 1525)(p=0.013)$. Testicular atrophy was found in 2 patients in the OR group $(0.2 \% ; 2 / 984)$ and $0 \%$ in the LPEC group $(0 / 1525 ; p=0.078)$. In addition, postoperative recurrence was $1.3 \%(13 / 1035)$ in the OR group and $0.5 \%(6 / 1182)$ in the LPEC group $(p=0.056)$ (Table 3). Intraoperatively, contralateral PPV was detected in 396 of 1014 (39\%) patients who were diagnosed with a clinically unilateral presentation. The contralateral PPV was confirmed by the evidence of insufflation in the groin through the PPV to the scrotum or labia, and all these patients underwent prophylactic surgery. As prophylactic surgery was related to $\mathrm{MClH}, \mathrm{MClH}$ was analyzed in patients who had a clinically unilateral inguinal hernia. The incidence of $\mathrm{MCIH}$ was 3.7\% (33/887) in the OR group and $0.3 \%(3 / 1014)$ in the LPEC group $(p<0.01)$ (Table 3). All MCIH and recurrence were re-operated laparoscopically. All patients in this study were discharged in 1 or 2 days.

\section{Discussion}

The laparoscopic approach has become increasingly popular as the techniques develop promptly. The advantages of this method, compared with open procedures, have been reported grossly in the literature [6-9]. Single port laparoscopic extraperitoneal inguinal hernia ligation in children offers many advantages over conventional open or three-port laparoscopic hernia repairs [6], cosmetic results are considered to be better in LPEC. Postoperative pain is reduced, and operative times are shortened. LPEC can also easily visualize the contralateral ring, and hence reduce the risk of metachronous hernia. More importantly, LPEC, due to lack of dissection of the spermatic vessels and the vas deferens, decreases the potential possibility of iatrogenic cryptorchidism and testicular atrophy [7].

This technique is a trivial modification to the established approaches of percutaneous ligation of the internal ring with the assistance of a laparoscope instead of a hooked apparatus in other similar reports (Fig. 1). It has been reported that needle- and needle-like apparatus could decrease the operating time and improve the cosmetic results by purse-stringing the internal ring [8]. Also, a two-hooked device would take less time than a single-hooked device $[10,11]$. However, in our cohort, surgical times averaged 17 minutes for unilateral hernia laparoscopically, only a slight increase compared to that of open repair, there was no statistically significant difference in unilateral hernia repair between the two groups. Surgical times averaged 22 minutes for bilateral LPEC group was much less than that of the routine open counterpart $(p<0.01)$.

Concerning the perioperative complications in this study, inguinal bleeding or hematoma occurred more frequently in the OR group than in the LPEC group. This issue was partly attributed to the extensive dissection of the cord structure in the OR group, which could be the cause of the testicular atrophy. Another issue was wound infection, which occurred similarly between the two groups. In our institution, the umbilicus was regularly cleaned before surgery, and the incision was placed circumferentially in the infra-umbilical crease, which could reduce the infection in the LPEC group (Table 3). 
Previously reported testicular complications in hernia sac ligation included testicular atrophy and iatrogenic cryptorchidism in male patients [12]. In previous reports, laparoscopic hernia repair was considered less invasive to the vas and vessels than OR, as the laparoscopic maneuver did not intervene in the structure of the inguinal canal. In our cohort, the incidence of testicular ascending in the LPEC group was statistically lower than that in the OR group. This fact supports the lower invasiveness of LPEC. However, long-term follow-up is required to evaluate the difference in testicular complications between these two approaches, but early results in our cohort are promising.

A previous study reported that one of the causes of recurrence was single ligation[13]. Double ligation was introduced to all patients in both groups to prevent the knots' loosening from a single ligation. The recurrence rate in LPEC was disputed in several reports[14,15]; however, LPEC in our cohort was acceptable from the perspective of recurrence $(p=0.056)$. In the OR group, the hernia sac may be damaged during the process of dissection, especially in male patients. This damage may lead to recurrence. On the other hand, in LPEC, the peritoneum could be injured and skipped when negotiating over the vas and vessels. Injury and skip were likely to contribute to recurrence. Complete circumferential ligation of the hernia sac is crucial to prevent a recurrence.

One of the advantages of LPEC repair is the prevention of $\mathrm{MCIH}$. In the LPEC group, $39 \%$ of patients with clinical unilateral hernia had confirmed contralateral PPV and underwent a prophylactic procedure. The incidence rate of $\mathrm{MCIH}$ was reduced from $3.7 \%$ in conventional repair to $0.3 \%$ in LPEC. Considering the lower incidence of $\mathrm{MCIH}$ in the LPEC group, prophylactic contralateral hernia repair in the LPEC group seems to be effective in preventing $\mathrm{MClH}$. This effectiveness of LPEC for preventing $\mathrm{MCIH}$ was supported by comparative studies $[4,7,16,17]$. A previous report stated that the contralateral PPV was common in pediatric patients, with a range of $20 \%-60 \%[16]$, open exploration is no longer recommended. The administration of contralateral PPV remains controversial since the clinical significance of an incidental finding of a PPV is still debated, and there is a concern for overtreatment. In this study, 3.7\% (33 patients of 887 with unilateral hernia) in the OR group developed $\mathrm{MClH}$. Considering that $39 \%$ of the patients in this group were hypothesized to have contralateral PPV referring to the incidence of LPEC group, only around $9.5 \%(33 / 346)$ of contralateral PPV in the OR group would develop $\mathrm{MClH}$. Therefore, for almost $91.5 \%$ of unilateral patients, prophylactic repair seems irrational and unnecessary. However, there is no concrete method to predict the percentage of patients with contralateral PPV who will develop MCIH at the current. Most of the parents requested to ligate the contralateral PPV to avoid possible secondary operations when consulting the benefit and risk of the surgery. In the LPEC operation, the asymptomatic contralateral internal ring was carefully checked, and when a PPV was confirmed, prophylactic surgery was performed regardless of the patency size. A prospective, randomized study with long-term follow-up is required to confirm this in the future.

As mentioned in this study, the surgical technique was modified to use a single needle without the assistance of the grasping forceps or the hollow hooked needle apparatus. Some may suspect that the learning curve of the procedure might influence the outcome of the operation. However, this is a simple manipulation similar to the reported laparoscopic percutaneous extraperitoneal hernia repair, except for 
the laparoscope assistance to negotiate the suture over the vas and the spermatic vessels. The experience and skill of the surgeon may affect the outcomes. All open operations are performed by previously experienced pediatric surgeons, while laparoscopic hernia repair does not require advanced skills and techniques. Actually, two surgeons in this study were trained to perform the procedure with basic proficiency in laparoscopy, and from our experience, 10 to 20 cases are sufficient to gain expertise. With this technique, we obtained excellent outcomes such as outstanding cosmesis, prompt operating time, no handling of the spermatic cord, and scarless wound healing. Our follow-up results also proved that laparoscope-assisted single-needle LPEC could be an alternative to conventional hernia repair to prevent $\mathrm{MClH}$.

However, this study also has limitations. This study is a short-term retrospective study conducted at a single institution. Concerns about $\mathrm{MClH}$ as well as testicular ascending or atrophy may need long-term follow-up for evaluation. Continued follow-up and accurate reports of recurrence are important to find the differences in these techniques. A randomized, prospective study with long-term follow-up is necessary in the future.

\section{Conclusions}

In this retrospective study, LPEC provides a minimally invasive approach with comparable results from the perspective of testicular complications and recurrent rates based on short-term outcomes. It is also argued that prophylactic surgery for contralateral hernia is beneficial for preventing $\mathrm{MCIH}$ without increased complication rate or operating time compared to the conventional open approach. Based on these data, it is recommended that laparoscope-assisted single-needle LPEC be used as an option to treat inguinal hernia in children.

\section{Abbreviations}

$\mathrm{OR}=$ open repair

LPEC = laparoscopic percutaneous extraperitoneal closure

$\mathrm{PPV}=$ patency processus vaginalis

$\mathrm{MCIH}=$ metachronous contralateral inguinal hernia

\section{Declarations}

\section{Ethics approval and consent to participate}

This retrospective chart review study involving human participants was in accordance with the ethical standards of the institutional and national research committee and with the 1964 Helsinki Declaration and its later amendments or comparable ethical standards. The Human Investigation Committee (IRB) of 
Shanghai Children's Medical Center approved this study. The present study was a retrospective study, and Informed consent was obtained from all patients' parents or guardians.

\section{Consent of publication:}

Written informed consent was obtained from the patients' parents or guardians for publication of this article and any accompanying images. A copy of the written consent is available for review by the Editor of this journal.

\section{Availability of data and materials}

The datasets used and/or analyzed during the current study are available from the corresponding author on reasonable request.

\section{Competing interests:}

The authors declare that they have no competing interests.

\section{Funding:}

No funding was received for the completion of this work.

\section{Authors Contributions}

SW made mainly contribution to the conception and design of the work, drafted the work and language editing. XX, RH collected the data and did the statistical analysis. HZ and LZ did the follow-up. JS was accountable for all aspects of the work, who were referred to when encountering the medical issues. All authors have read and approved the manuscript.

Acknowledgments: not applicable.

\section{References}

1. Disma N, Withington D, McCann ME, et al. Surgical practice and outcome in 711 neonates and infants undergoing hernia repair in a large multicenter RCT: Secondary results from the GAS Study. J Pediatr Surg 201853 (9):1643-1650. doi:10.1016/j.jpedsurg.2018.01.003

2. Silber $S$, Becker VM, Seufert R, Muensterer OJ. Fertility in males after childhood, adolescent, and adult inguinal operations. J Pediatr Surg 201954 (1):177-183. doi:10.1016/j.jpedsurg.2018.10.012

3. Shibuya S, Miyazaki E, Miyano G, et al. Comparison of laparoscopic percutaneous extraperitoneal closure versus conventional herniotomy in extremely low birth weight infants. Pediatr Surg Int 2019 35 (1):145-150. doi:10.1007/s00383-018-4386-2

4. Nakashima $\mathrm{M}$, Ide $\mathrm{K}$, Kawakami $\mathrm{K}$ Laparoscopic versus open repair for inguinal hernia in children: a retrospective cohort study. Surg Today 201949 (12):1044-1050. doi:10.1007/s00595-019-01847-0 
5. Chen Y, Wang F, Zhong H, Zhao J, Li Y, Shi Z. A systematic review and meta-analysis concerning single-site laparoscopic percutaneous extraperitoneal closure for pediatric inguinal hernia and hydrocele. Surg Endosc 201731 (12):4888-4901. doi:10.1007/s00464-017-5491-3

6. Korkmaz M, Guvenc BH. Comparison of Single-Port Percutaneous Extraperitoneal Repair and ThreePort Mini-Laparoscopic Repair for Pediatric Inguinal Hernia. J Laparoendosc Adv Surg Tech A 2018 28 (3):337-342. doi:10.1089/lap.2016.0223

7. Chong AJ, Fevrier HB, Herrinton LJ. Long-term follow-up of pediatric open and laparoscopic inguinal hernia repair. J Pediatr Surg 201954 (10):2138-2144. doi:10.1016/j.jpedsurg.2019.01.064

8. Davies DA, Rideout DA, Clarke SA. The International Pediatric Endosurgery Group Evidence-Based Guideline on Minimal Access Approaches to the Operative Management of Inguinal Hernia in Children. J Laparoendosc Adv Surg Tech A. 2017 doi:10.1089/lap.2016.0453

9. Baradaran N, Wood CM, McCoy OO, Prasad MM, Stec AA. Laparoscopic intra-abdominal patent processus vaginalis ligation in pediatric urology practice. J Pediatr Urol 201713 (5):512 e511512.e516. doi:10.1016/j.jpurol.2017.03.026

10. Yonggang H, Changfu Q, Ping W, et al. Single-port laparoscopic percutaneous extraperitoneal closure of inguinal hernia using "two-hooked" core needle apparatus in children. Hernia. 2019 doi:10.1007/s10029-019-01933-9

11. Li S, Liu X, Wong KKY, Liu L, Li Y. Single-port laparoscopic herniorrhaphy using a two-hooked cannula device with hydrodissection. J Pediatr Surg 201853 (12):2507-2510. doi:10.1016/j.jpedsurg.2018.08.009

12. Celebi S, Yildiz A, Ucgul A, et al. Do open repair and different laparoscopic techniques in pediatric inguinal hernia repairs affect the vascularization of testes? J Pediatr Surg 201247 (9):1706-1710. doi:10.1016/j.jpedsurg.2012.03.044

13. Amano $\mathrm{H}$, Tanaka $\mathrm{Y}$, Kawashima $\mathrm{H}$, et al. Comparison of single-incision laparoscopic percutaneous extraperitoneal closure (SILPEC) and open repair for pediatric inguinal hernia: a single-center retrospective cohort study of 2028 cases. Surgical endoscopy 201731 (12):4988-4995. doi:10.1007/s00464-017-5472-6

14. Shalaby R, Abd Alrazek M, Elsaied A, et al. Fifteen Years Experience with Laparoscopic Inguinal Hernia Repair in Infants and Children. J Laparoendosc Adv Surg Tech A 201828 (1):101-105. doi:10.1089/lap.2017.0269

15. Darmawan KF, Sinclair T, Dunn JCY. Comparison of laparoscopic and open pediatric inguinal hernia repairs at two institutions. Pediatr Surg Int 201834 (12):1293-1298. doi:10.1007/s00383-018-4360-z

16. Kokorowski PJ, Wang HH, Routh JC, Hubert KC, Nelson CP. Evaluation of the contralateral inguinal ring in clinically unilateral inguinal hernia: a systematic review and meta-analysis. Hernia 201418 (3):311-324. doi:10.1007/s10029-013-1146-z

17. Shalaby R, Elsayaad I, Alsamahy 0 , et al. One trocar needlescopic assisted inguinal hernia repair in children: a novel technique. J Pediatr Surg. 2017 doi:10.1016/j.jpedsurg.2017.08.020 

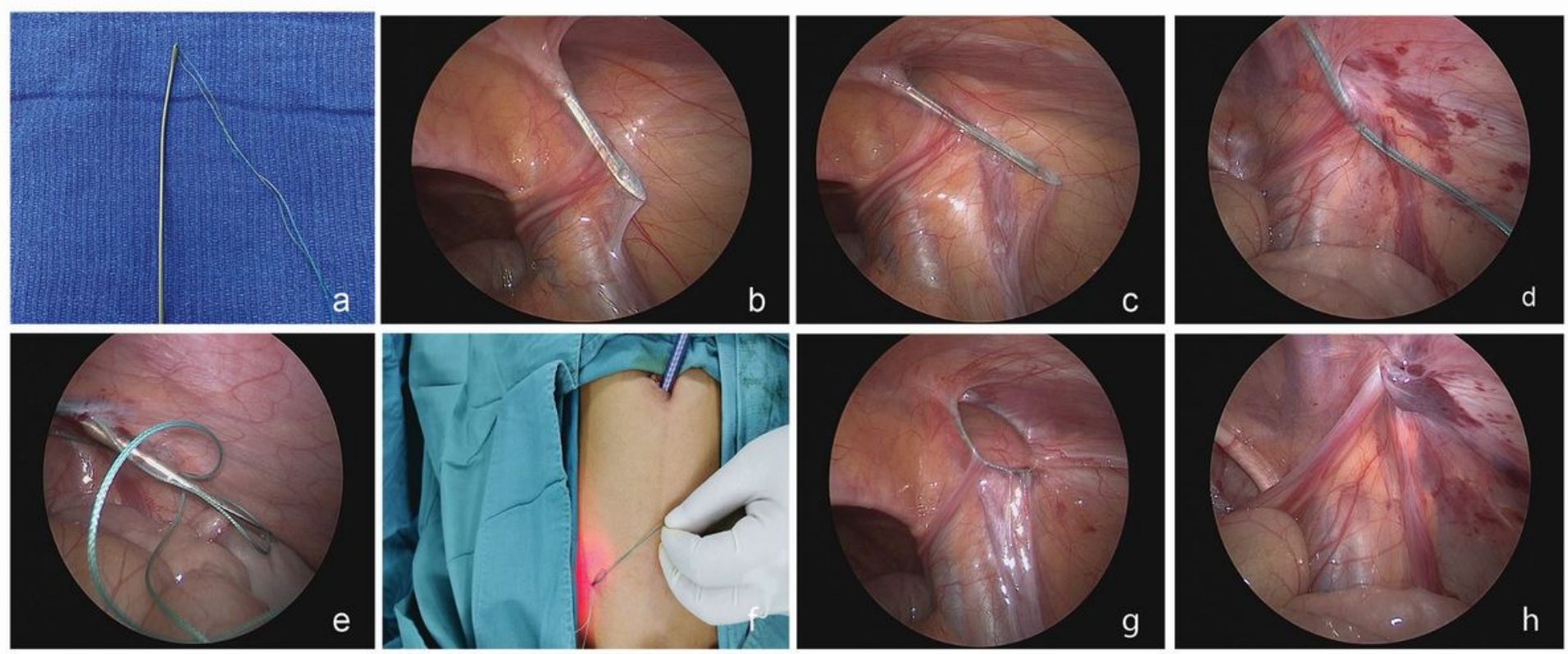

\section{Figure 1}

$<p>$ LPEC procedure in right side inguinal hernia for a male patient $</ p><p>$ \&nbsp;a) Needle tips with eyelet for passing a 2-0 polyester suture; b-d) Medial aspect dissection of the internal ring by negotiating over the vas and vessels with the needle,\&nbsp;the first suture loop was left in the abdomen with the assistance of camera feeding the loop; e-g) Lateral advancing to feed the previous loop and the first loop used as a lasso by pulling the extracorporeal end to create purse ring around the internal ring;

h)\&nbsp;Ligation of the internal ring $</ p>$ 